\title{
The Postbiotics, Totipro PE0401, and Probiotic Mixture, PF1001, Modulate the Gut Microbiota and Ameliorate Diarrhea in Weaning Piglets
}

\author{
Hsieh Hsun Ho*, Yi Wei Kuo, Jui Fen Chen, Yu Fen Huang, Cheng Ruei Liu, Jia Hung Lin, and \\ Ching Wei Chen
}

Research and Development Department, Glac Biotech Co., Ltd., Tawian

*Corresponding author: Hsieh Hsun Ho, Research and Development Department, Tainan City 744, Taiwan

\section{ARTICLE INFO}

Received: May 05, 2020

Published: 蔧 May 29, 2020

Citation: Hsieh Hsun Ho, Yi Wei Kuo, Jui Fen Chen, Yu Fen Huang, Cheng Ruei Liu. The Postbiotics, Totipro PE0401, and Probiotic Mixture, PF1001, Modulate the Gut Microbiota and Ameliorate Diarrhea in Weaning Piglets. Biomed J Sci \& Tech Res 28(1)-2020. BJSTR. MS.ID.004584.

Abbreviations: TGE: Transmissible Gastroenteritis; ZnO: Zinc Oxide; CTAB: Cetyltrimethylammonium Bromide; SDS: Sodium Dodecyl Sulphate; NA: Nutrient Agar; PCA: Plate Count Agar; IgA: Immunoglobulin A; IgG: Immunoglobulin G; MRSA: Methicillin-Resistant S. Aureus

\begin{abstract}
Most piglets are weaned between three and four weeks of age, which is very soon after birth. In spite of its stress, early weaning is nowadays a common practice to increase the productive yield of pig farms. Diarrhea is a frequent challenge in weaning piglets and the dehydration caused by severe diarrhea can easily result in death. Antibiotics and medicinal zinc oxide were commonly used to treat the diarrhea; however, concerns of drug resistance and heavy metal pollution have made these treatments arguable. Therefore, in this study, supplements of the postbiotics, Totipro PE0401, or/and the mixture of ten probiotic strains, PF1001, are investigated for relieving the symptom and promoting the health in weaning piglets. Multiple combinations of Totipro PE0401 or/and PF1001 were added to the daily feed for six weeks during the weaning period. Better feed conversion rates and less odor of feces were seen in all Totiproor/and PF1001-treated groups. The composition of gut microbiota was modulated, and immune response was enhanced toward a better defense against pathogens. The improvement on diarrhea was comparable with the antibiotic-treated group. In conclusion, the supplement of the postbiotics, Totipro PE0401, together with probiotic mixture, PF1001, is an alternative solution to weaning diarrhea in piglets.
\end{abstract}

Keywords: Probiotics; Postbiotics; Diarrhea; Weaning; Piglets

\section{Introduction}

Pigs are a popular form of livestock, with high economic values in food, clothing, cosmetics, and medical industries. More than one billion pigs butchered each year worldwide, and the main consuming countries are in Asia as dietary food source. The weaning stage is considered one of the most critical periods in swine production, where piglet performance can be seriously affected and where they are predisposed to the overgrowth of opportunistic pathogens [1]. Piglet diarrhea or "scour" can be common at both the neonatal and the post-weaning stages. It is a common cause of mortality and is often closely associated with poor hygiene, inappropriate husbandry (e.g., early weaning), stressful environment and inappropriate feeding factors.

Poor hygiene can lead to overgrowth of pathogenic bacteria in the environment. Escherichia coli (E. coli) strains are common within the first week of life, and again in the first week after weaning. The weaning process increases the stress on piglets, and thus their susceptibility to viral and bacterial infections. Diarrhea in these older piglets tends to be less severe, and mortality rates lower than in pre-weaning piglets. Potential sources of diarrhea at this stage include E. coli, Rotavirus, TGE (Transmissible gastroenteritis), salmonellosis, Campylobacter and Brachyspira hyodysenteriae [2]. Therefore, uses of antibiotics or medications are almost inevitable during the weaning process in the past. However, due to the increasing problem of drug resistance of bacteria, the use of antibiotic growth promoters for prevention of diarrheal diseases in piglets has been banned since 2006 [3]. Coincidentally, due to the concern of environmental and food safety, the use of medicinal zinc oxide ( $\mathrm{ZnO}$ ) must be phased out by 2022 [4]. Thus, alternative strategies are urgent needs to prevent diarrhea and promote the health in weaner piglets. Various natural 
materials such as probiotics, prebiotics, organic acids, and plant extracts have been tested as effective alternatives to antibiotics [3]

Recently, it has been shown that weaning stress not only changed microbial composition and function, but also altered the microbial metabolic profiles in the intestine [5]. Gut microbiota is symbiotically residing in digestive tracts, and fecal microbiota transplantation are often used to regulate gut microbial colonization [6]. In human, supplements of probiotics have been recommended in pediatric gastrointestinal diseases, and considered for prevention of antibiotic associated, Clostridium difficile-induced, or nosocomial diarrhea [7]. Therefore, postbiotic or probiotic products might provide a new insight in alleviating weaning stress and facilitating disease prevention during the period of weaning in piglets $[8,9]$. In this study, effects of not only the mixture of ten probiotic strains,PF1001, but also the postbiotics, Totipro PE0401, on the growth and health in weaning piglets were evaluated in 4-week old weaning piglets.

\section{Materials and Methods}

All experiments were performed in accordance with approved guidelines. The animal protocol was approved by the Agricultural Technology Research Institute. (Project number AAI24-E-P10708).

\section{In vitro Cell Growth Evaluation}

Every generation of probiotic or pathogenic bacteria strains was incubated in $5 \mathrm{ml}$ MRS $(0.05 \%$ cysteine added if necessary) broth at $37^{\circ} \mathrm{C}$ for $17 \mathrm{hrs}$. The third generation was spun down and re-suspended in fresh broth as the activated culture. For the growth assay of probiotic strains, the cell concentration was adjusted to O.D. $600=1$ as the starting point of this test. $3 \mathrm{ml}$ of bacteria was added into $12 \mathrm{ml}$ HMO (Human Milk Oligosaccharide) or Totipro PE0401 solutions and incubated at $37^{\circ} \mathrm{C}$ for $6 \mathrm{hrs}$. Bacteria was spun down and re-suspended in fresh broth. O.D.600 was measured as the finishing point of this test. The growth was measured by the comparison between values of 0.D.600 at starting and finishing points. For the growth assay of pathogenic strains, $100 \mu \mathrm{l}$ of activated culture was added to $4.9 \mathrm{ml}$ postbiotic or Totipro PE0401 solutions, and incubated at $37^{\circ} \mathrm{C}$ for $48 \mathrm{hrs}$. Three adjacent tenfold serial dilutions of plate count were performed and numbers of bacteria colonies were compared between treated and untreated groups.

\section{Preparation of Supplementation}

The dry product of Totipro (postbiotics product number PE0401) and probiotic mixture (probiotics product number PF1001) were prepared and provided by glac Biotech Co., Ltd. (Tainan City, Taiwan). Totipro PE0401 was the fermentation of four probiotic strains: Lactobacillus plantarum, Bifidobacterium longum subsp. infantis, Lactobacillus acidophilus and Lactobacillus salivarius subsp. salicinius. Heat-killed cell counts of Totipro PE0401 was $>1 \times 10^{7} \mathrm{CFU} / \mathrm{g}$. The chemical composition of Totipro PE0401 was list as Table 1. Commercial strains used in pathogenic inhibition assay were obtained from their origins, and their postbiotics were collected in-house: Lactobacillus rhamnosus GG, Lactobacillus acidophilus LA-5, and Bifidobacterium animalis subsp. Lactis BB$12^{\circ}$ were from Chr. Hansen, Milwaukee, WI. Lactobacillus gasseri OLL2716 (LG-21) was from Meiji, Tokyo, Japan. Bifidobacterium longum BB536 was from Morinaga, Tokyo, Japan.

Probiotic mixture PF1001 contains ten strains of lactic acid bacteria, (Bifidobacterium animalis subsp. lactis) CP-9, (Bifidobacterium breve) Bv-889, (Bifidobacterium longum subsp. infantis) BLI-02, (Lactobacillus acidophilus) TYCA06, (Lactobacillus casei) CS-773, (Lactobacillus paracasei) GL-156, (Lactobacillus plantarum) LPL28, (Lactobacillus reuteri) GL-104, (Lactobacillus rhamnosus) F-1 and (Streptococcus thermophiles) SY-66. Viable cell counts of probiotic mixture PF1001 was $1 \times 10^{11} \mathrm{CFU} / \mathrm{g}$. The powder was suspended in fodder before feeding. Six groups were set up as below: vehicle, Tylosin $(0.05 \%)$, Totipro $(0.2 \%)$, Totipro $(0.1 \%)$, Totipro $(0.1 \%)$ together with PF1001 (1x10 $\mathrm{CFU} / \mathrm{g})$ and PF1001 alone $\left(1 \times 10^{7} \mathrm{CFU} / \mathrm{g}\right)$.

Table 1: Chemical composition of Totipro PE0401.

\begin{tabular}{|c|c|}
\hline $\begin{array}{c}\text { Chemical Composition of LFB } \\
\text { Power }\end{array}$ & Per 100 g Contribution \\
\hline Calories & $333.6 \mathrm{Kcal}$ \\
\hline Crude protein & $12.8 \mathrm{~g}$ \\
\hline Crude fat & $1.2 \mathrm{~g}$ \\
\hline Saturated fat & $0.05 \mathrm{~g}$ \\
\hline Carbohydrate & $67.9 \mathrm{~g}$ \\
\hline Sugar & $9.360 \mathrm{~g}$ \\
\hline Glucose & $0.681 \mathrm{~g}$ \\
\hline Maltose & $0.844 \mathrm{~g}$ \\
\hline Lactose & $5.011 \mathrm{~g}$ \\
\hline Sodium & $4247.5 \mathrm{mg}$ \\
\hline Lactic acid & $8.874 \mathrm{~g}$ \\
\hline
\end{tabular}

\section{Animals and Experimental Design}

One hundred and forty-four crossbred ((Landrace x Yorkshire) $x$ Duroc) 4 week-old, weaning piglets with an average initial body weight of $8.68 \pm 0.04 \mathrm{~kg}$ participated in the study. Weaning piglets were randomly allotted one of 6 treatments (with 4 replicates of 6 piglets per stall) in a completely randomized design. The experimental diets consisted of a basal diet with no treatment as vehicle, a basal diet contained $0.05 \%$ antibiotic (Tylosin $0.05 \%$ ), a basal diet contained $0.2 \%$ Totipro PE0401 (Totipro 0.2\%), a basal diet contained $0.1 \%$ Totipro PE0401 (Totipro 0.1\%), a basal diet contained $0.1 \%$ Totipro PE0401 together with PF1001 (1x10 $\mathrm{CFU} / \mathrm{g})$, and a basal diet contained PF1001 (1x107 CFU/g) alone. Diets (Tables $2 \& 3$ ) were formulated to meet or exceed the nutrient requirements recommended by the National Research Council (Nutrient Requirements for Swine, 2012). 
Food and water were offered ad libitum throughout the experimental period. The body weight, daily feed consumption, daily weight gain, diarrhea incidence analysis and feed conversion ratio were recorded every 2 week. After 6 weeks of feeding, the serum of weaning piglets was collected for immunity analysis (IgA and IgG). The stool was collected for fecal microbiota analysis and odor analysis.

Table 2: Composition of basal diets.

\begin{tabular}{|c|c|c|}
\hline \multirow{2}{*}{ Ingredient, g/kg } & \multicolumn{2}{|c|}{ Basal Diets } \\
\cline { 2 - 3 } & Week 0-2 & Week 3-6 \\
\hline Corn, yellow & 492.5 & 577.5 \\
\hline Soybean meal-CP48 & 180.0 & 180.0 \\
\hline Soybean oil & 40.0 & 30.0 \\
\hline Cooked corn & 100.0 & 50.0 \\
\hline Full fat soy flour & 100.0 & 100.0 \\
\hline Whey powder & 50.0 & 25.0 \\
\hline Vitamin,Mineral & 2.0 & 2.0 \\
\hline Amino acid & 5.5 & 5.5 \\
\hline Choline chloride & 1.0 & 1.0 \\
\hline CaCO $_{3}$ & 11.0 & 11.0 \\
\hline Phosphorus & 14.0 & 14.0 \\
\hline Salt & 4.0 & 4.0 \\
\hline
\end{tabular}

Table 3: Chemical composition of basal diets.

\begin{tabular}{|c|c|c|}
\hline \multirow{2}{*}{ Chemical Composition } & \multicolumn{2}{|c|}{ Basal Diets } \\
\hline & Week 0-2 & Week 3-6 \\
\hline Water (\%) & 9.73 & 10.75 \\
\hline Crude protein (\%) & 17.40 & 17.38 \\
\hline Crude fat (\%) & 7.32 & 5.06 \\
\hline Crude fiber (\%) & 3.25 & 5.61 \\
\hline Crude ash (\%) & 6.98 & 6.73 \\
\hline Total phosphorus (\%) & 0.77 & 0.76 \\
\hline Calcium (\%) & 1.14 & 1.25 \\
\hline Energy (cal / g) & 4020.720 & 3843.330 \\
\hline ASP.(\%) & 1.969 & 1.569 \\
\hline Thr.(\%) & 1.008 & 0.758 \\
\hline Ser.(\%) & 0.995 & 0.796 \\
\hline Glut.(\%) & 3.621 & 2.886 \\
\hline Gly.(\%) & 0.825 & 0.643 \\
\hline Ala.(\%) & 0.991 & 0.791 \\
\hline Cyst.(\%) & 0.243 & 0.216 \\
\hline Val.(\%) & 0.943 & 0.721 \\
\hline Meth.(\%) & 0.397 & 0.396 \\
\hline Isol.(\%) & 0.847 & 0.638 \\
\hline Leu.(\%) & 1.706 & 1.330 \\
\hline Tyr.(\%) & 0.794 & 0.613 \\
\hline Pheny.(\%) & 0.758 & 0.709 \\
\hline Lys.(\%) & 1.483 & 1.280 \\
\hline Try.(\%) & 0.186 & 0.224 \\
\hline Hist.(\%) & 0.501 & 0.352 \\
\hline Arg.(\%) & 1.359 & 1.032 \\
\hline
\end{tabular}

\section{Diarrhea Incidence Analysis}

Diarrhea incidence was recorded daily in each stall throughout the entire experiment through direct observation conducted by 2 evaluators. The diarrhea was determined based on fecal consistency using a modification of the method described by a previous study [10]. The feces were ranked on the following scale: 0 = solid; 1 = semi-solid; 2 = semi-liquid; and 3 = liquid. The piglets were considered to have diarrhea when the fecal consistency was level 2 or 3, as described previously by Cheng et al. [11] and calculated as follows: Diarrhea incidence $(\%)=$ [the number of pigs with diarrhea in each stall / total numbers of pigs / days] x $100 \%$.

\section{Bacterial DNA Extraction and 16S rRNA Sequencing}

Before the start and the designated end point, fecal specimens were collected from all subjects using the DNA / RNA Shield ${ }^{\text {TM }}$ reagent in the fecal collection tube (Zymo Research Corp, California, USA). Each collection tube (with a spoon attached to the lid) is prefilled with DNA/RNA Shield ${ }^{\mathrm{TM}}$ (9 mL). Bacterial DNA was extracted using cetyltrimethylammonium bromide / sodium dodecyl sulphate (CTAB / SDS) method, and the obtained nucleic acids (DNA and RNA) were stored under freezing $-80{ }^{\circ} \mathrm{C}$ for subsequent analysis. The DNA purity was determined with a ratio of OD 260 to OD 280 in the range of $1.8 \sim 2.0$. Using specific primers $341 \mathrm{~F}$ (F, forward primer; 5'-CCTAYGGGRBGCASCAG-3') and 806R (R, reverse primer; 5'GGACTACNNGGGTATCTAAT-3') to amplify the highly variable V3-V4 regions of the bacterial 16S rRNA gene by PCR Zone. A sample preparation kit (Illumina, San Diego, California, United States) without TruSeq DNA PCR was used to construct a double-ended library (each sample insertion size was 450-470 bp). The amplified DNA sizing was checked by TapeStation (Agilent Technologies). High-throughput sequencing was performed on the Illumina HiSeq2500 platform. The sequences generated went through a filtering process to obtain the qualified reads. Total reads were merged, removed low-quality sequence, removed chimera sequence and clustered the OTU at $97 \%$ similarity with the Greengenes database. All OTU sequences and diversity analysis were using CLC Microbial Genomics Module (Qiagen, Germany), basespace (illumine, USA) and Graphpad prism 7 (Graphpad Software, USA). A $p$ value less than 0.05 is considered statistically significant.

\section{6s Sequencing of Fecal Sample}

Fecal samples were collected at ZT18 being equal to PM 2:00 and all specimens were extracted using Qiagen DNA kit according to the manufacturer's instructions. DNA purity was determined with a ratio of OD 260 to 280 in the range of 1.8 2.0. 16S rDNA PCR used metagenomic DNA as template and amplified with the bacterial-specific primers S17 (5' TCGTCGGCAGCGTCAGATGT GTATAAGAGACAGCCTACGG GNGGCWGCAG 3') and A21 (5' GTCTCGTGGGCTCGGAGATG TGTATAAGAGACAGGACTAC HVGGGTATCTAATCC 3'). The amplified DNA sizing was checked by TapeStation (Agilent Technologies). Sequencing was carried out by Illumina Miseq platform. DNA samples were attached indices and Illumina sequencing adapters using the Nextera XT Index Kit. After library construction, 
samples were mixed with MiSeq Reagent Kit v3 (600-cycle) and loaded onto a Miseq cartridge, then onto the instrument. Automated cluster generation and a $2 \times 300$ bp paired-end sequencing run was performed. The sequences generated went through a filtering process to obtain the qualified reads. Total reads were merged, removed low-quality sequence, removed chimera sequence and clustered the OTU at $97 \%$ similarity with the Greengenes database. All OTU sequences and diversity analysis were using CLC Microbial Genomics Module (Qiagen, Germany), basespace (illumine, USA) and Graphpad prism 7 (Graphpad Software, USA). A $p$ value less than 0.05 is considered statistically significant.

\section{Statistical Analysis}

Data were analyzed using one-way ANOVA through the GLM procedure in SAS software (Version 9.4; SAS Institute, Cary, NC, USA). The results are expressed as mean \pm SEM. Means were compared using Duncan's multiple range test at a significance level of ${ }^{*} \mathrm{p}<0.05,{ }^{* *} \mathrm{p}<0.01,{ }^{* * *} \mathrm{p}<0.001$.

\section{Fecal Microbiological Analysis}

Fecal samples of weaning piglets were plated in triplicate on NA (Nutrient agar), PCA (plate count agar) and MRS with $0.05 \%$ cysteine agar broth for detecting Escherichia coli, total gut bacteria and Lactic acid bacteria (Lactobacillus and Bifidobacterium) respectively. CFUs on each plate were evaluated to study microbiota in gut of weaning piglets.

\section{Odor Analysis}

The fecal of weaning piglets $250 \mathrm{~g}$ was collected in the sample bag for ferment 30 minutes. The odor analysis was detected by Gastec GV-100 machine, injected $100 \mathrm{ml}$ above gas into the machine for 30 secs. Identify the odor concentration $\left(\mathrm{NH}_{3}, \mathrm{~N}_{2} \mathrm{~S}\right.$ and R-SH) based on color change.

\section{Immunity Analysis of Weaning Piglets (IgA and IgG)}

IgA and IgG concentrations in serum samples were evaluated using Pig IgA and IgG ELISA kit (abcam, ab190536 and ab190813). Each sample was analyzed in triplicate.

\section{Results}

\section{In vitro Growth Assay}

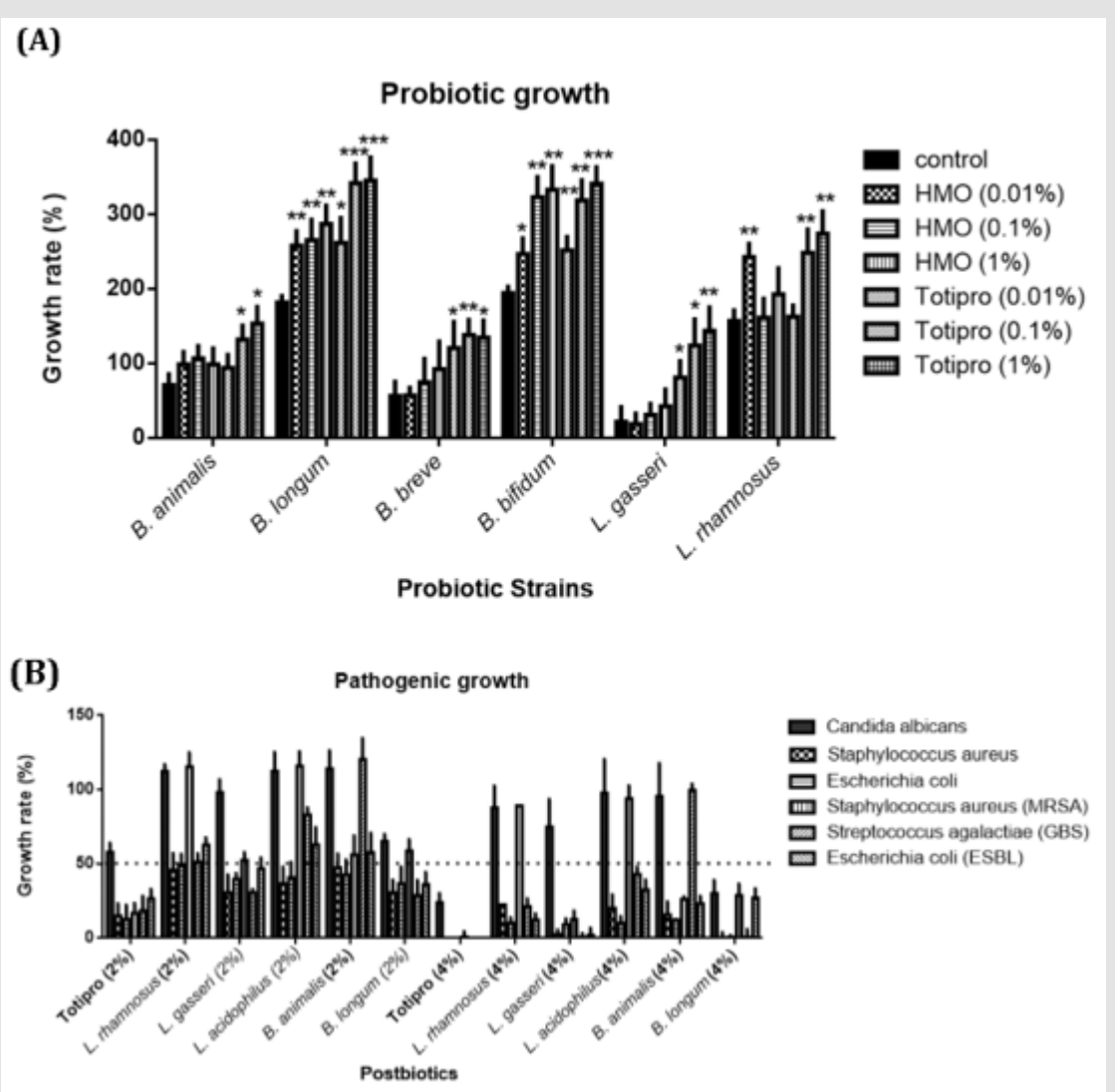

Figure 1: In vitro growth effects of Totipro on probiotic and pathogenic strains.

(A) Effects of HMO (0.01\%-1\%) and Totipro (0.01\%-1\%) on cell growth in six probiotic bacteria strains. $\mathrm{N}=3$ in each groups. ${ }^{*} \mathrm{P}<0.05 ;{ }^{* *} \mathrm{P}<0.01 ;{ }^{* *} \mathrm{p}<0.001$ vs. control group.

(B) Effects of Totipro and five other postbiotics on cell growth in six pathogenic bacteria strains. The dotted line represented the $50 \%$ growth inhibition. 
The postbiotics, Totipro PE0401, showed different effects on growth rates of probiotic and pathogenic bacteria (Figure 1). Of the probiotic strain, $B$. animalis, the growth rate increased significantly $\left({ }^{*} \mathrm{p}<0.05\right)$ in $0.1 \%$ and $1 \%$ Totirpo solutions (Figure 1A). Of $B$. longum, the growth rate increased significantly $\left({ }^{*} \mathrm{p}<0.05\right.$, ${ }^{* *} \mathrm{p}<0.01,{ }^{* * *} \mathrm{p}<0.001$ ) in all HMO (Human Milk Oligosaccharide) and Totipro solutions. Of $B$. breve, the growth rate increased significantly $\left({ }^{*} \mathrm{p}<0.05,{ }^{* *} \mathrm{p}<0.01\right)$ in all three Totipro solutions. Of $B$. bifidum, the growth rate increased significantly $\left({ }^{*} \mathrm{p}<0.05,{ }^{* *} \mathrm{p}<0.01\right.$, *** $\mathrm{p}<0.001$ ) in all HMO (Human Milk Oligosaccharide) and Totipro solutions. Of $L$. gasseri, the growth rate increased significantly $\left({ }^{*} \mathrm{p}<0.05,{ }^{* *} \mathrm{p}<0.01\right)$ in all three Totipro solutions. Of L. rhamnosus, the growth rate increased significantly $\left({ }^{* *} \mathrm{p}<0.01\right)$ in $0.01 \% \mathrm{HMO}$, $0.1 \%$ Totipro, and $1 \%$ Totipro solutions (Figure $1 \mathrm{~A}$ ).

Of pathogenic strain, C. albicans, the $50 \%$ growth inhibition was seen only in $4 \%$ Totipro and $4 \%$ postbiotic solutions of $B$. longum (Figure 1B). 85\% growth inhibitions of $S$. aureus and $E$. coli were seen in $2 \%$ Totipro solution, while only $50 \%$ growth inhibitions were seen in other $2 \%$ postbiotic solutions. Better growth inhibitions of $S$. aureus and E. coli were seen in 4\% Totipro and $4 \%$ postbiotic solutions of L. gasseri and B. longum than in other postbiotic solutions. Of Methicillin-Resistant S. aureus (MRSA), an $83 \%$ growth inhibition was seen in $2 \%$ Totipro solution, while $50 \%$ growth inhibitions were not achieved in other $2 \%$ postbiotic solutions. More than $70 \%$ growth inhibitions of MRSA were seen in $4 \%$ Totipro and other $4 \%$ postbiotic solutions except for solutions of L. rhamnosus and L. acidophilus. Of S. agalactiae (Group
B Streptococcus, GBS) and high drug-resistant E. coli (extendedspectrum $\beta$-lactamases, ESBL), $81 \%$ and $73 \%$, respectively, growth inhibitions were seen in $2 \%$ Totipro solution, while $50 \%$ growth inhibitions were not achieved in $2 \%$ postbiotic solutions of $L$. rhamnosus, L. acidophilus, and B. animalis. A more complete growth inhibition was seen in $4 \%$ Totipro solution, while the $50 \%$ growth inhibition of GBS was still not achieved in 4\% postbiotic solution of B. animalis (Figure 1B).

\section{Growth Performance}

Body weights of piglets were similar without significant differences in all groups on week 0. A significant difference was seen on week 6 in group $\mathrm{E}$, and the body weight was significantly higher $(29.14 \pm 0.95 \mathrm{~kg})$ than in group A $(27.43 \pm 0.58 \mathrm{~kg})$ (Figure $2 \mathrm{~A})$. Moreover, daily weight gains were analyzed every two weeks, and results showed significantly $\left({ }^{*} \mathrm{P}<0.05\right)$ higher weight gains in group $\mathrm{C}, \mathrm{D}$ and $\mathrm{E}(0.60 \pm 0.05,0.59 \pm 0.04$ and $0.60 \pm 0.02 \mathrm{~kg}$, respectively) than in group A $(0.51 \pm 0.04 \mathrm{~kg})$ during week 5-6 (Figure 2B). Daily food consumptions were measured every two weeks, and significantly $\left({ }^{*} \mathrm{P}<0.05\right)$ less food consumptions were seen in group $\mathrm{C}$ and $\mathrm{E}(1.00 \pm 0.06$, and $0.97 \pm 0.07 \mathrm{~kg}$, respectively) than in group $\mathrm{A}$ $(1.17 \pm 0.10 \mathrm{~kg}$ ) (Figure 2C). Furthermore, the feed conversion rate in group C, D and E $(1.69 \pm 0.12,1.67 \pm 0.18$ and $1.63 \pm 0.11$, respectively) during week 5-6 were significantly $\left({ }^{* *} \mathrm{P}<0.01, * \mathrm{P}<0.05\right)$ lower than in group A (2.30 \pm 0.28$)$ (Figure 2D). In conclusion, supplements of $0.1 \%$ Totipro (group C) and $0.2 \%$ Totipro (group E) displayed the best feed conversion rate.

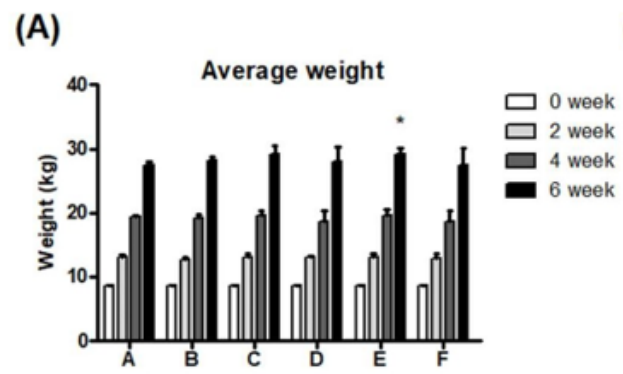

(B)

(C)

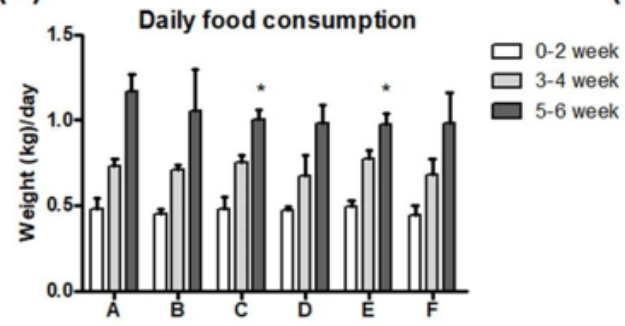

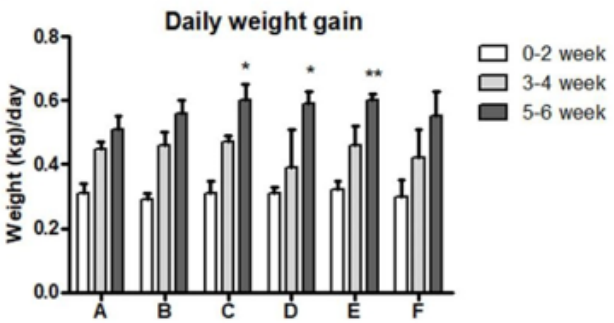

(D)

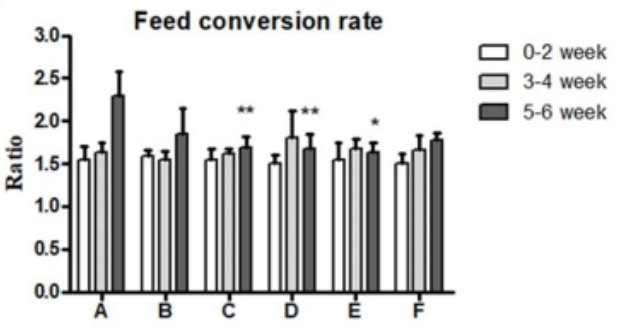

Figure 2: Effects of different dietary supplements on growth performance of weaning piglets.

(A) Average weight,

(B) Daily weight gain,

(C) Daily food consumption,

(D) Feed conversion rate. A: Vehicle; B: 0.05\% Tylosin; C: $0.2 \%$ Totipro; D: 0.1\% Totipro; E: 0.1\% Totipro together with PF1001 $\left(1 \times 10^{7} \mathrm{CFU} / \mathrm{g}\right)$; F: PF1001 ( $\left.1 \times 10^{7} \mathrm{CFU} / \mathrm{g}\right) . \mathrm{N}=24$ in each groups. ${ }^{*} \mathrm{P}<0.05 ;{ }^{* *} \mathrm{P}<0.01$ vs. group A. 


\section{Fecal Odor}

The main sources of odor are ammonia, hydrogen sulphide and thiol derivatives in feces. After additions of multiple combinations of Totipro PE0401 or/and PF1001 for 6 weeks, levels of ammonia were significantly $\left({ }^{*} \mathrm{p}<0.05\right)$ lower in group $C$ and $E(2.39 \pm 1.46$; $2.35 \pm 0.94 \mathrm{ppm}$, respectively) than in group A (4.45 $\pm 1.11 \mathrm{ppm})$, as shown in (Figure $3 \mathrm{~A}$ ) Levels of hydrogen sulphide were significantly $\left({ }^{*} \mathrm{P}<0.05,{ }^{* *} \mathrm{P}<0.01\right)$ lower in group $\mathrm{C}(20.58 \pm 11.30 \mathrm{ppm}), \mathrm{D}$
(22.37 $\pm 7.41 \mathrm{ppm}), \mathrm{E}(17.06 \pm 3.34 \mathrm{ppm})$ and $\mathrm{F}(21.44 \pm 13.24 \mathrm{ppm})$ than in group A $(36.49 \pm 14.05 \mathrm{ppm})$, of which group $\mathrm{E}$ displayed the most reduction (Figure 3B). Levels of thiol derivatives were significantly $\left({ }^{*} \mathrm{P}<0.05\right)$ lower in group $\mathrm{C}(0.72 \pm 0.52 \mathrm{ppm}), \mathrm{D}$ $(0.73 \pm 0.49 \mathrm{ppm})$ and $\mathrm{E}(0.60 \pm 0.57 \mathrm{ppm})$ than in group $\mathrm{A}(1.46 \pm 0.67$ ppm) (Figure 3C). Among these gases, hydrogen sulphide is most odorous component, and the supplement of $0.1 \%$ Totipro together with PF1001 $\left(1 \times 10^{7} \mathrm{CFU} / \mathrm{g}\right)$ is sufficient on decreasing the fecal odor.
(A)

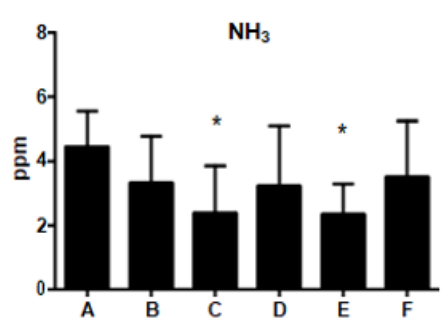

(B)

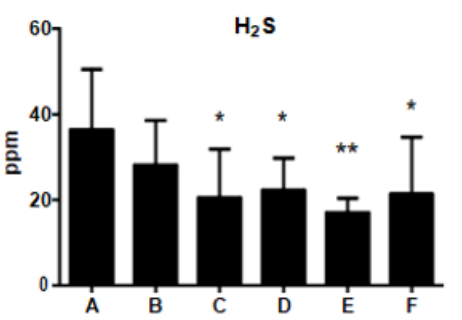

(C)

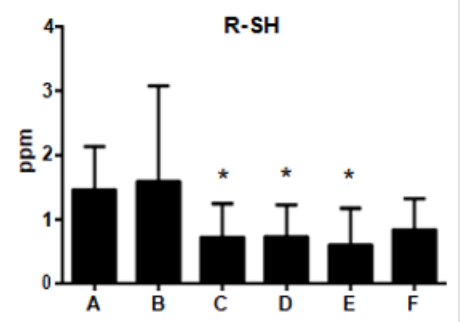

Figure 3: Effects of different dietary supplements for 6 weeks on fecal odor of weaning piglets.

(A) Ammonia,

(B) Hydrogen sulfide,

(C) Thiol derivatives. A: Vehicle; B: 0.05\% Tylosin; C: $0.2 \%$ Totipro; D: 0.1\% Totipro; E: 0.1\% Totipro together with PF1001 $\left(1 \times 10^{7} \mathrm{CFU} / \mathrm{g}\right)$; F: PF1001 $\left(1 \times 10^{7} \mathrm{CFU} / \mathrm{g}\right) . \mathrm{N}=24$ in each groups. ${ }^{*} \mathrm{P}<0.05 ;{ }^{* *} \mathrm{P}<0.01$ vs. group A.

\section{Cytokine Levels}

(A)

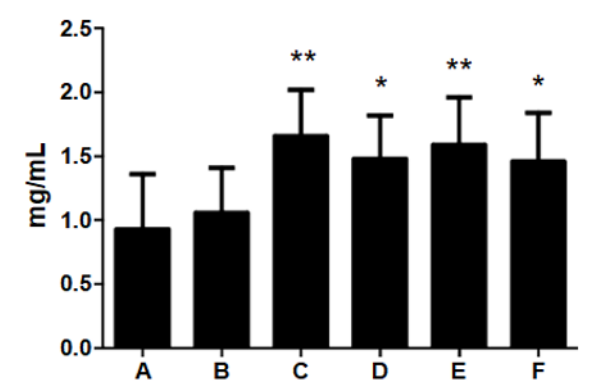

(B)

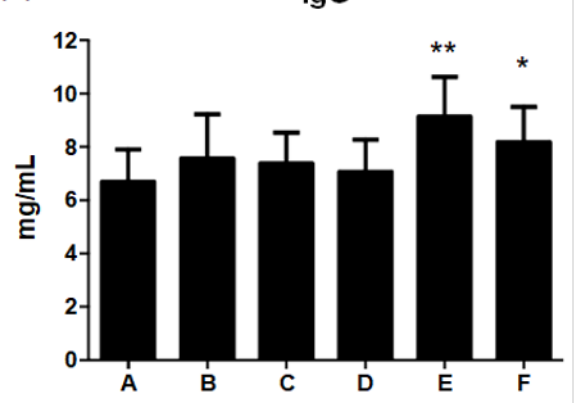

Figure 4: Effects of different dietary supplements for 6 weeks on cytokine levels of weaning piglets.

(A) IgA,

(B) IgG. A: Vehicle; B: 0.05\% Tylosin; C: 0.2\% Totipro; D: 0.1\% Totipro; E: 0.1\% Totipro together with PF1001 (1×107 CFU/g);

F: PF1001 $\left(1 \times 10^{7}\right.$ CFU/g). $\mathrm{N}=24$ in each groups. * $\mathrm{P}<0.05 ; * * \mathrm{P}<0.01$ vs. A group.

Contents of IgA and IgG were immune indicators for pathogenic defense in piglets. Levels of $\operatorname{IgA}$ were significantly $\left({ }^{* *} \mathrm{P}<0.01,{ }^{*} \mathrm{P}\right.$ $<0.05)$ higher in group C, D, E, and F $(1.66 \pm 0.36 ; 1.48 \pm 0.34 ; 1.59 \pm 0.37$; $1.46 \pm 0.38 \mathrm{mg} / \mathrm{mL}$, respectively) than in group A $(0.93 \pm 0.43 \mathrm{mg} /$ $\mathrm{mL}$ ), as shown in (Figure 4A). Furthermore, levels of IgG were significantly higher in groups $\mathrm{E}(9.15 \pm 0.38 \mathrm{mg} / \mathrm{mL}, \mathrm{P}<0.01)$ and $\mathrm{F}$ $(8.19 \pm 1.32 \mathrm{mg} / \mathrm{mL}, \mathrm{P}<0.05)$ than in group $\mathrm{A}(6.69 \pm 1.22 \mathrm{mg} / \mathrm{mL})$; (Figure 4B). The result indicated the supplement of $0.1 \%$ Totipro together with PF1001 $\left(1 \times 10^{7} \mathrm{CFU} / \mathrm{g}\right)$ significantly enhanced IgA and IgG contents.

\section{Fecal Microbiota Analysis}

In order to elucidate effects of different dietary supplements on gut microbiota, compositions of lactic acid bacteria, E. coli and total bacteria were compared between week 0 and 6 . Levels of lactic acid bacteria were significantly ( ${ }^{* *} \mathrm{p}<0.01$ ) higher in group $\mathrm{C}, \mathrm{D}$ and $\mathrm{E}$ on week 6 ( $9.55 \pm 0.31 ; 9.12 \pm 0.16$; and $9.58 \pm 0.38 \mathrm{CFU} / \mathrm{mL}$, respectively) than on week $0(8.09 \pm 0.49 ; 7.96 \pm 0.75$; and $7.92 \pm 0.55 \mathrm{CFU} / \mathrm{mL}$, respectively), as shown in (Figure $5 \mathrm{~A}$ ). Moreover, levels of diarrheacausing bacteria, E. coli, were significantly ( $\left.{ }^{* * *} \mathrm{P}<0.001, * \mathrm{P}<0.05\right)$ lower in group B, C, D, E and F on week $6(6.00 \pm 0.28$; 6.81 \pm 0.43 ; 
$7.02 \pm 0.29 ; 6.15 \pm 0.37$ and $6.68 \pm 0.42 \mathrm{CFU} / \mathrm{mL}$, respectively) than on week $0(7.74 \pm 0.41 ; 7.98 \pm 0.36 ; 7.86 \pm 0.42 ; 7.84 \pm 0.22$; and $7.46 \pm 0.12$ $\mathrm{CFU} / \mathrm{mL}$, respectively), of which group $\mathrm{C}$ and $\mathrm{E}$ displayed the best inhibitions (Figure 5B). On the other hand, the level of total bacteria in each group showed no significant difference between week 0 and 6 (Figure 5C). These results indicated the supplement of $0.1 \%$ Totipro together with PF1001 $\left(1 \times 10^{7} \mathrm{CFU} / \mathrm{g}\right)$ significantly increased the number of lactic acid bacteria and reduced the number of $E$. coli with comparable efficiency to Tylosin $0.05 \%$ treatment in weaning piglets.
(A)

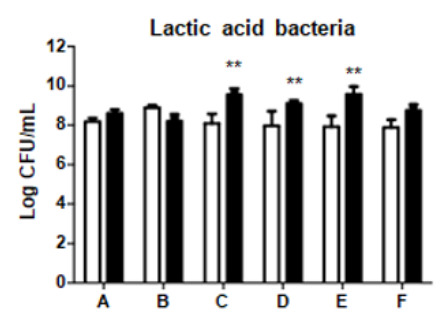

(B)

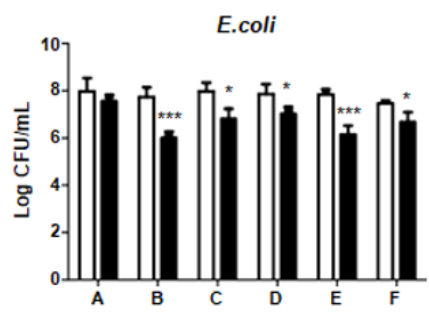

(C)

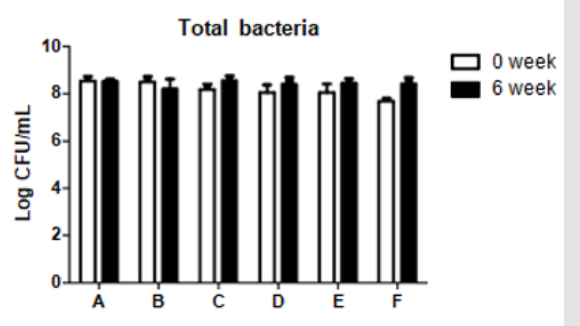

Figure 5: Comparisons of different dietary supplements on fecal microbiota of weaning piglets.

(A) Lactic acid bacteria,

(B) E. coli,

(C) Total bacteria. A: Vehicle; B: 0.05\% Tylosin; C: 0.2\% Totipro; D: 0.1\% Totipro; E: $0.1 \%$ Totipro together with PF1001 $\left(1 \times 10^{7}\right.$ $\mathrm{CFU} / \mathrm{g})$; F: PF1001 (1×107 CFU/g).

$\mathrm{N}=24$ in each groups. ${ }^{*} \mathrm{P}<0.05 ;{ }^{* *} \mathrm{P}<0.01 ;{ }^{* * *} \mathrm{P}<0.001$ vs. 0 week.

\section{Diarrhea Ratio}

After additions of multiple combinations of Totipro PE0401 or/ and PF1001 in the feed, occurrences of diarrhea were significantly $\left({ }^{* * *} \mathrm{P}<0.001,{ }^{* *} \mathrm{P}<0.01\right)$ lower on week $2-3$ in group $\mathrm{B}, \mathrm{C}, \mathrm{D}, \mathrm{E}$ and
F $(7.89 \pm 1.44 ; 1.19 \pm 1.37 ; 4.83 \pm 0.59 ; 1.61 \pm 1.12$ and $4.35 \pm 3.98 \%$, respectively) than in group A (14.21 $\pm 1.15 \%)$, as shown in Figure 6 . By the end of the treatment on week 5-6, already no occurrence of diarrhea in group B, C, D, E and F, while $4.23 \pm 1.21 \%$ of diarrhea ratio was still seen in group $\mathrm{A}$.

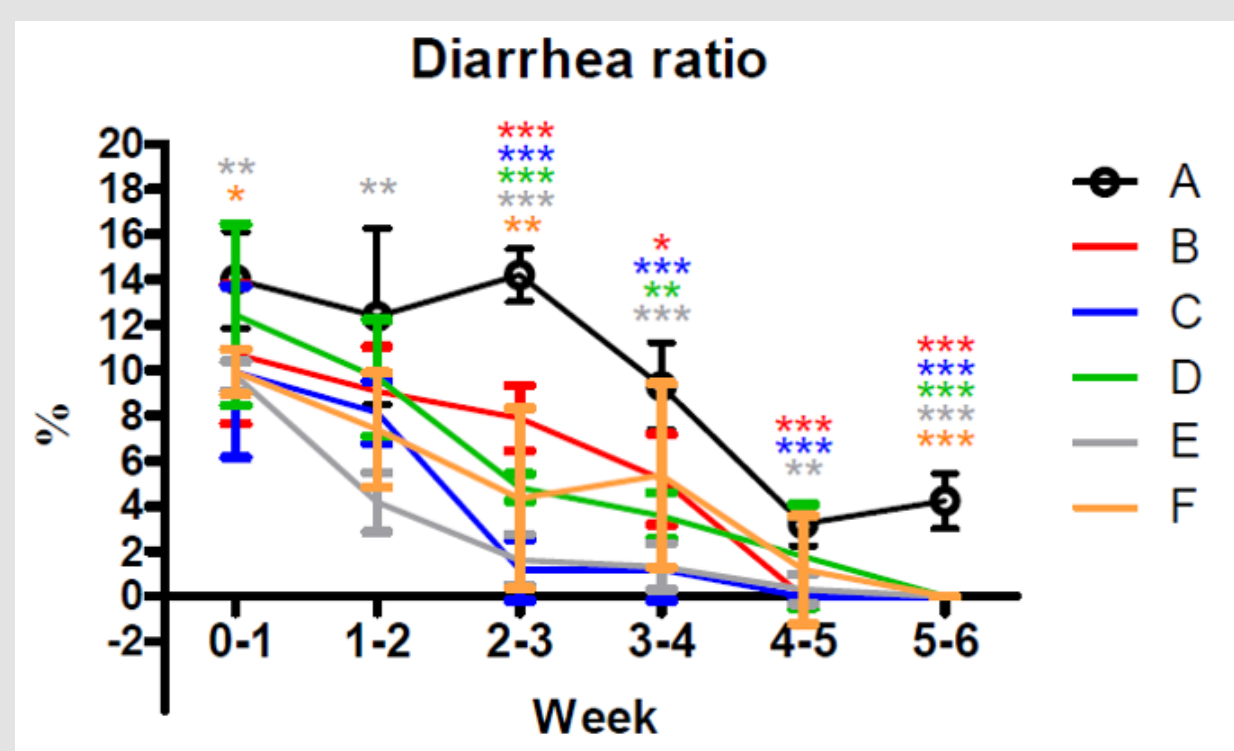

Figure 6: Comparisons of different dietary supplements on weekly diarrhea ratio of weaning piglets. A: Vehicle (black); B: 0.05\% Tylosin (red); C: $0.2 \%$ Totipro (blue); D: 0.1\% Totipro (green); E: 0.1\% Totipro together with PF1001 (1×107 CFU/g) (gray); F: PF1001 (1×107 CFU/g) (orange). $\mathrm{N}=24$ in each groups. ${ }^{*} \mathrm{P}<0.05 ;{ }^{* *} \mathrm{P}<0.01$; ${ }^{* *} \mathrm{P}<0.001$ vs. A group.

\section{NGS Analysis on the Fecal Microbiota}

Fecal samples were collected before treatments started on week 0 and at the end of the study on week 6 . Fecal microbiota compositions were analyzed using the 16S rRNA genes and several dramatic changes of the microbial ecology were observed in groups treated with Totipro PE0401 or/and PF1001. On the level of genus (Figure 7A \& Table 4), numbers of Lactobacillus, Streptococcus,
Bifidobacterium and Akkermania were all richer in groups supplied with Totipro or/and PF1001 than in vehicle group. Ratios of Streptococcus, Bifidobacterium and Akkermania were richer in groups supplied with Totipro $(0.2 \%)$ and Totipro $(0.1 \%)$ together with PF1001 than in Tylosin (0.05\%) group. In addition, levels of pathogenic bacteria, Clostridium, Staphylococcus, Helicobacter and Escherichia were lower in the group supplied with Totipro together with PF1001. 
The change of fecal microbiota was further analyzed on the level of species (Figure 7B \& Table 5). Among the family of Lactobacillus, abundances of Lactobacillus taiwanensis, Lactobacillus johnsonii, and Lactobacillus delbrueckii were higher in groups supplied with Totipro or/and PF1001 than in the vehicle group. A significant $\left({ }^{*} \mathrm{p}<0.05\right)$ decrease of Lactobacillus taiwanensis (by 0.479 fold) was seen only in the group supplied with Totipro $(0.1 \%)$ together with PF1001. Significant $\left({ }^{*} \mathrm{p}<0.05,{ }^{* *} \mathrm{p}<0.01\right)$ increases of Lactobacillus johnsonii were seen in groups supplied with Tylosin $(0.05 \%)$, Totipro $(0.2 \%)$, Totipro $(0.1 \%)$, Totipro $(0.1 \%)$ together with PF1001, and PF1001 only (by 28.375, 44.797, 13.568, 426.056 and 266.884 fold, respectively). Significant $\left({ }^{*} \mathrm{p}<0.05,{ }^{* *} \mathrm{p}<0.01\right)$ increases of Lactobacillus delbrueckii were seen in groups supplied with Totipro $(0.2 \%)$ 、 Totipro $(0.1 \%)$ together with PF1001, and PF1001 only (by 1.245, 1.284 and 7.427 fold, respectively).
A

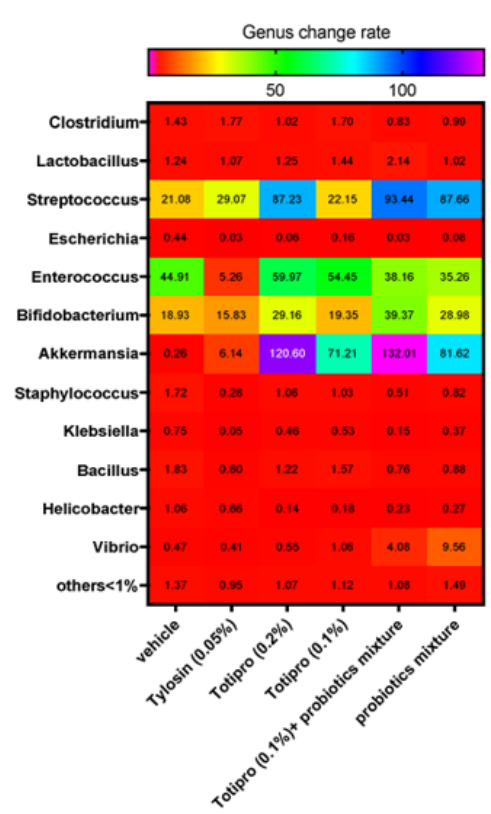

B

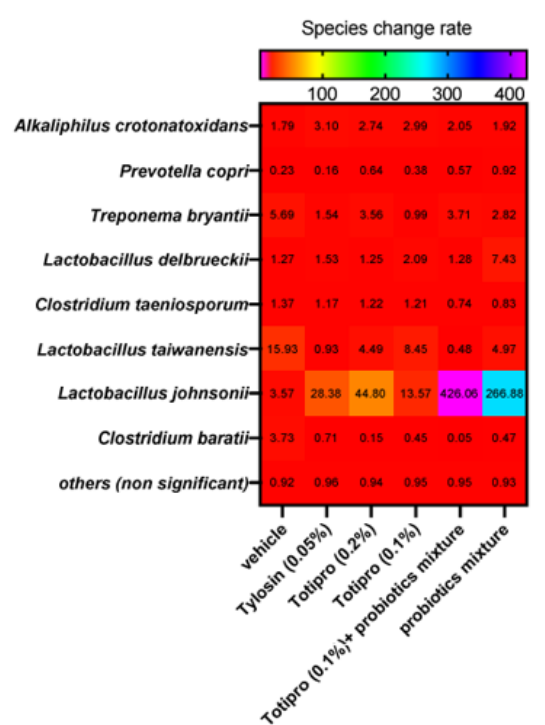

Figure 7: Heat map of fecal microbiota change rates in different dietary supplemented groups. Fecal samples were collected before treatments started on week 0 and at the end of the study on week 6. Change rates were analyzed on levels of genus (A) and species (B). Data are expressed as mean for $n=24$ weaning piglets per group.

Table 4: Lists of fecal microbiota change rates on the level of genus.

\begin{tabular}{|c|c|c|c|c|c|c|c|c|c|c|c|c|}
\hline & \multicolumn{6}{|c|}{ Change Rate } & \multicolumn{6}{|c|}{ t-Test } \\
\hline & Vehicle & $\begin{array}{l}\text { Tylosion } \\
(0.05 \%)\end{array}$ & $\begin{array}{l}\text { Totipro } \\
(0.2 \%)\end{array}$ & $\begin{array}{l}\text { Totipro } \\
(0.1 \%)\end{array}$ & $\begin{array}{c}\text { Totipro } \\
(0.1 \%)+ \\
\text { Probiotics } \\
\text { mixture }\end{array}$ & $\begin{array}{l}\text { Probiotics } \\
\text { mixture }\end{array}$ & Vehicle & $\begin{array}{l}\text { Tylosin } \\
(0.05 \%)\end{array}$ & $\begin{array}{l}\text { Totipro } \\
(0.2 \%)\end{array}$ & $\begin{array}{l}\text { Totipro } \\
(0.1 \%)\end{array}$ & $\begin{array}{c}\text { Totipro } \\
(0.1 \%)+ \\
\text { Probiotics } \\
\text { mixture }\end{array}$ & $\begin{array}{l}\text { Probiotics } \\
\text { mixture }\end{array}$ \\
\hline Clostridium & 1.43 & 1.77 & 1.02 & 1.7 & 0.83 & 0.99 & 1 & 0.099 & 0.6884 & 0.133 & 0.0617 & 0.7365 \\
\hline Lactobacillus & 1.24 & 1.07 & 1.25 & 1.44 & 2.14 & 1.02 & 1 & 0.586 & 0.8419 & 0.614 & 0.2487 & 0.4241 \\
\hline Streptococcus & 21.08 & 29.07 & 87.23 & 22.15 & 93.44 & 87.66 & 1 & 0.602 & 0.2091 & 0.837 & 0.7913 & 0.968 \\
\hline Escherichia & 0.44 & 0.03 & 0.06 & 0.16 & 0.03 & 0.08 & 1 & 0.422 & 0.0517 & 0.104 & 0.8794 & 0.4865 \\
\hline Enterococcus & 44.91 & $5.26^{*}$ & 59.97 & 54.45 & $38.16^{* *}$ & 35.26 & 1 & 0.032 & 0.9085 & 0.054 & 0.0065 & 0.0635 \\
\hline Bifidobacterium & 18.93 & 15.83 & 29.16 & 19.35 & 39.37 & 28.98 & 1 & 0.606 & 0.0581 & 0.148 & 0.5987 & 0.4647 \\
\hline Akkermansia & 0.26 & 6.14 & 120.6 & 71.21 & 132.01 & 81.62 & 1 & 0.361 & 0.7421 & 0.077 & 0.0505 & 0.6284 \\
\hline Staphylococcus & 1.72 & 0.28 & 1.06 & 1.03 & 0.51 & 0.82 & 1 & 0.426 & 0.5632 & 0.944 & 0.7336 & 0.7562 \\
\hline Klebsiella & 0.75 & 0.05 & 0.46 & 0.53 & 0.15 & 0.37 & 1 & 0.327 & 0.2992 & 0.225 & 0.3549 & 0.3129 \\
\hline Bacillus & 1.83 & 0.6 & 1.22 & 1.57 & 0.76 & 0.88 & 1 & 0.073 & 0.5733 & 0.376 & 0.9521 & 0.6185 \\
\hline Heliocobacter & 1.06 & 0.66 & 0.14 & 0.18 & 0.23 & 0.27 & 1 & 0.506 & 0.8061 & 0.081 & 0.7211 & 0.4902 \\
\hline Vibrio & 0.47 & 0.41 & 0.55 & 1.06 & 4.08 & 9.56 & 1 & 0.678 & 0.4847 & 0.962 & 0.8425 & 0.4955 \\
\hline Others $<1 \%$ & 1.37 & 0.95 & 1.07 & 1.12 & 1.08 & 1.49 & & & & & & \\
\hline
\end{tabular}

Note: Data are expressed as mean of fecal microbiota change rate for $n=24$ weaning piglets per group. Values with different superscript letters are significantly different at ${ }^{*} \mathrm{p}<0.05 ;{ }^{* *} \mathrm{p}<0.01$ by t-test. 


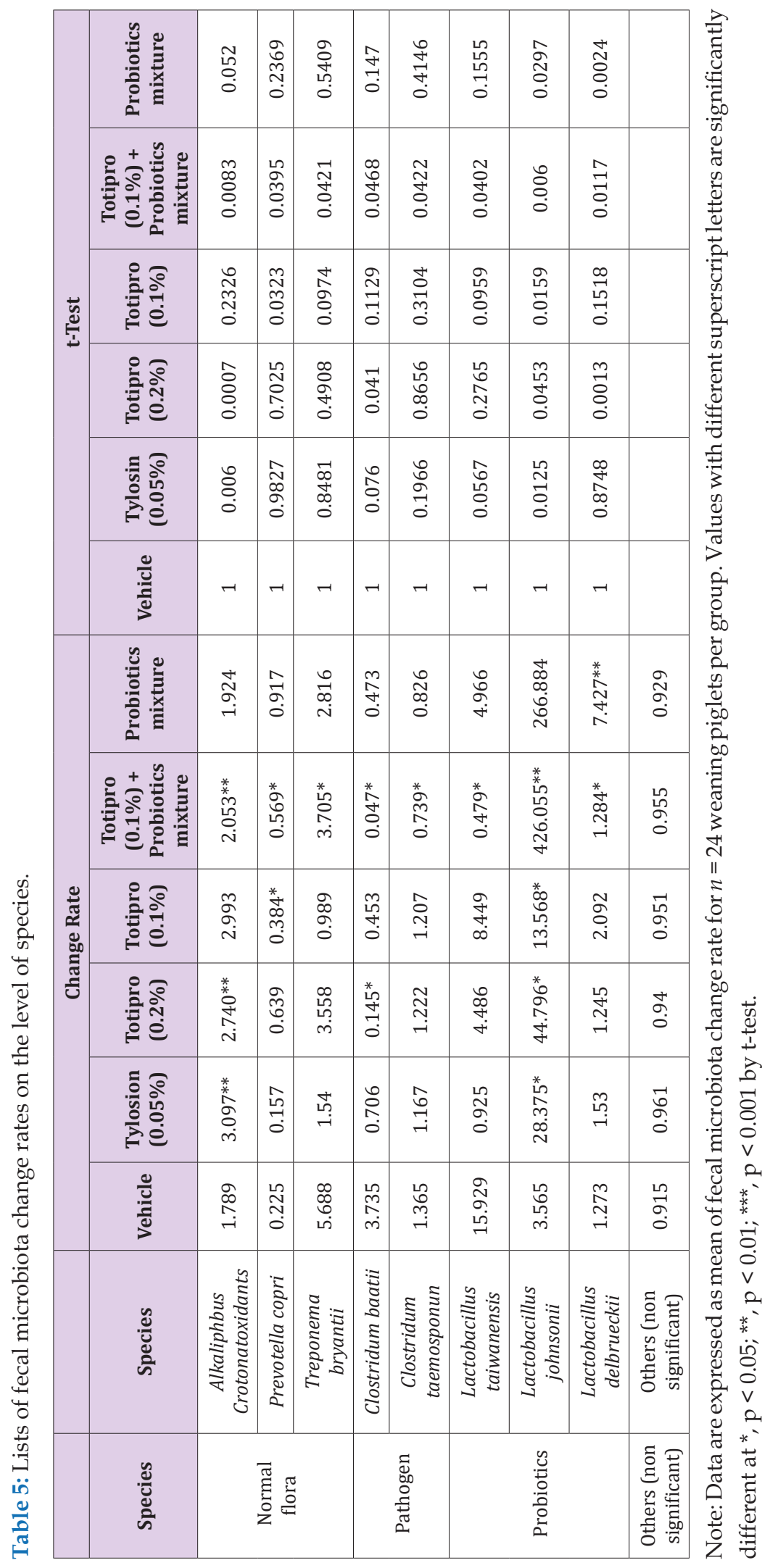


Among pathogenic species, significant $\left({ }^{*} p<0.05\right)$ decreases of Clostridium baratii were seen in groups supplied with Totipro $(0.2 \%)$ and Totipro $(0.1 \%)$ together with PF1001 (by 0.145 and 0.047 fold, respectively). A significant $\left({ }^{*} \mathrm{p}<0.05\right)$ decrease of Clostridium taeniosporum was only seen in the group supplied with Totipro (0.1\%) together with PF1001 (by 0.739 fold). In general, supplements of Totipro or/and PF1001 have a trend of increasing beneficial bacteria and reducing pathogenic bacteria. Over all, the supplement of Totipro PE0401 together with PF1001 showed the best effect on increasing the richness of beneficial bacteria and inhibiting harmful gut microbiota significantly $(\mathrm{p}<0.05)$.

\section{Discussion}

From the result of our growth assay in vitro, the postbiotics of four probiotics, Totipro PE0401, show notable characteristics which promote the growth in probiotic strains and inhibit the growth in pathogenic strains. HMO (Human Milk Oligosaccharide) can be consumed and promote the growth in some probiotic strains [12]. Our result indicates a better capability of Totipro on promoting the growth of probiotic strains, and moreover, potentially enhancing the beneficial effects of them. Recently, the antibiotic activity of postbiotics has attracted much attention, and become the potential alternatives of antibiotics [13]. Our result demonstrates the effect of Totipro on growth inhibition of pathogenic strains, and the potential of Totipro on the antibiotic effect by itself. Different from probiotics, Totipro contains no live bacteria, and is able to avoid the risk of administrating live microorganisms. If the infection of bacterial relocation is concerned, the use of Totipro is more suitable in newborn animals [14]. The better shelf life and economical properties of Totipro also provide another ideal type of food supplementary for stock industry; therefore, effects of Totipro alone or together with probiotics on health worth further investigations in vivo.

Weaning is a sudden, complex and highly stressful event in pig's life. It is a transition from liquid to solid food, and needs to be taken smoothly step by step to make sure the health of the piglet is in a good condition. The starter feed is very crucial to a successful transition and the taste is important to piglets. They have to like what they are eating to continue to return to the feeder [15]. From our results of daily weight gain, daily food consumption, and feed conversion rate, additions of Totipro or/and PF1001 do not affect the daily food consumption significantly, while the daily weight gain is actually increased comparing to the control and antibiotic groups. In other words, supplements of Totipro PE0401 or/and PF1001 are acceptable on taste and able to provide a better feed conversion rate in weaning piglets.

Intensive pig farms are by far the most popular, due to their potential to raise a large amount of pigs in a very cost-efficient manner. Inevitably, this kind of farming comes together with heavy feces and waste in a close area. The odor can spread widely and freely through air and is very unpleasant to the neighbourhood.
From our odor analysis of the feces, components of ammonia, hydrogen sulphide, and thiol derivatives are reduced upon the addition of Totipro or/and PF1001 in the feeding. The nitrogenand sulphur-containing gas contributes the most to the stinky smell of feces. Therefore, supplements of Totipro PE0401 or/and PF1001 decrease the production of strong odors and improve the environment of the piglet housing.

If the composition of gut microbiota is roughly divided into two ends of beneficial or pathogenic populations, the balance can be dynamically affected by the external condition of the host. Gut bacterial dysbiosis has emerged as a leading cause of post-weaning diarrhea [16], and treatment with capsulized fecal microbiota transplantation is proved to ameliorate the symptom in piglets [6]. From the analysis of fecal microbiota, additions of Totipro PE0401 or/and PF1001 do not affect the size but the proportion of microbiota. Take two populations as the example, the change of proportion showed an increase in the population of Lactic acid bacteria, which represents the beneficial bacteria, and a decrease in the population of Escherichia, which represents the major pathogenic bacteria which causes diarrhea.

The NGS analysis shows the change of microbiota community in a more comprehensive aspect [17]. The bacteria culture on petri dish grows all lactic acid bacteria, and shows an increase of the community upon the treatment. The richness of Lactobacillus, but not Bifidobacterium, in NGS analysis increases significantly, and the increase is contributed especially by the specie of $L$. johnsonii. The population of pathogenic bacteria, Escherichia, decreases significantly on petri dish, although the richness of the bacteria shows only a moderate, but not significant, reduction in NGS analysis. On the other hand, richness of another pathogenic bacteria, Clostridium, decreases significantly upon the supplement of Totipro PE0401 together with PF1001 in NGS analysis. Taken all together, our treatments modulate the microbiota toward a more beneficial composition in weaning piglets.

In piglets, the amount of immunoglobulin A $(\operatorname{IgA})$ is associated with the intestinal mucosal immunity against pathogens, and is especially important during weaning when a new form of feed is introduced [18]. The level of immunoglobulin G (IgG) represents the anti-infection immunity and is strongly associated with the survival of piglets [19]. Recent evidences have demonstrated that some probiotics facilitate increasing the host sIgA levels and some probiotics contribute to increase the host serum IgG levels [20]. Supplements of Totorpo or/and PF1001 elevate the level of IgA, and especially the supplement of Totipro together with PF1001 increases the level of IgG. This result indicates a fundamental enhancement of intestinal immunity is achieved by either Totipro or PF1001 supplements. Preferably, a more comprehensive protection feasible when Totipro PE0401 and PF1001 are given together.

Weaning diarrhea is one of the most frequent causes of heavy economic losses in pig herds. Although the antibiotic treatment was 
sufficient to solve this single problem, it raised more environmental issues and was limited for this use. Our previous study has demonstrated that the fermentation products of lactic acid bacteria have significant antibacterial activity against fish pathogens. Diet supplementation with lactic acid bacteria fermentation products in aquaculture significantly alters the gut microbiome of fish. In this study, supplements of Totipro or/and PF1001 reduce the occurrence of diarrhea, and stabilize the gut health sooner than the antibiotic treatment. Preferably, the best alleviation of the symptom is performed when Totipro and PF1001 are given together. One of the important characteristics of probiotics is the antibacterial activity, which is possibly achieved through the production of bacteriocins [21]. Moreover, researches about postbiotic applications on diarrhea in children have obtained positive effects [9], so as our results in piglets. Taken all together, a combination of the postbiotics, Totipro PE0401, together with probiotic mixture, PF1001, is able to modulate gut microbiota and alleviate diarrhea sufficiently in weaning piglets.

\section{Conclusion}

The supplement of the postbiotics, Totipro PE0401, together with probiotic mixture, PF1001, is an alternative solution to weaning diarrhea in piglets. Supplements of Totipro PE0401 or PF1001, or especially together, give better feed conversion rates and less odor of feces. The composition of gut microbiota was modulated, and immune response was enhanced toward a better defense against pathogens. The improvement on diarrhea were comparable with the antibiotic treatment.

\section{Acknowledgements}

We sincerely thank Dr. Lin, Chuan-Shun in Agricultural Technology Research Institute for handling treatments of piglets and data collections.

\section{Conflict of Interest}

All authors have declared that no support, financial or otherwise, has been received from any organization that may have an interest in the submitted work.

\section{References}

1. Knecht D, Cholewinska P, Jankowska Makosa A, Czyz K (2020) Developmentof Swine's Digestive Tract Microbiota and Its Relation to Production Indices-A Review. Animals (Basel) 10(3)

2. Bohl EH (1978) Rotavirus as a cause of diarrhea in pigs. J Am Vet Med Assoc 172(4): 458-463.

3. Vondruskova HSR, Trckova M, Zraly Z, Pavlik I (2010) Alternatives to antibiotic growth promoters in prevention of diarrhoea in weaned piglets: a review. Veterinarni Medicina 55(5): 26.

4. Satessa GD (2020) Effects of alternative feed additives to medicinal zinc oxide on productivity, diarrhoea incidence and gut development in weaned piglets. Animal 1-9.
5. Li Y, Yong Guo, Zhengshun Wen, Xuemei Jiang, Xin Ma, et al. (2018) Weaning Stress Perturbs Gut Microbiome and Its Metabolic Profile in Piglets. Sci Rep 8(1): 18068.

6. Tang W (2020) Capsulized faecal microbiota transplantation ameliorates post-weaning diarrhoea by modulating the gut microbiota in piglets. Vet Res 51(1): 55 .

7. Cameron D, Quak Seng Hock, Musal Kadim, Neelam Mohan, Eell Ryoo, et al. (2017) Probiotics for gastrointestinal disorders: Proposed recommendations for children of the Asia-Pacific region. World J Gastroenterol 23(45): 7952-7964.

8. Barba Vidal E, Martin Orue SM, Castillejos L (2018) Review: Are we using probiotics correctly in post-weaning piglets? Animal 12(12): 2489-2498.

9. Wegh CAM, Geerlings SY, Knol J, Roeselers G, Belzer C, et al. (2019) Postbiotics and Their Potential Applications in Early Life Nutrition and Beyond. Int J Mol Sci 20(19): 4673.

10. Cappai MG, Wolf P, Rust P, Pinna W, Kamphues J, et al. (2013) Raw hulled shredded acorns from Downy Oak (Quercus pubescens) in the diet of pigs: effects on digestibility and faeces characteristics. J Anim Physiol Anim Nutr (Berl) 97 Suppl 1: 1-5.

11. Cheng YH, Su D, Horng YB, Yu YH (2019) Effects of soybean meal fermented by Lactobacillus species and Clostridium butyricum on growth performance, diarrhea incidence, and fecal bacteria in weaning piglets. Annals of Animal Science.

12. Thongaram T, Hoeflinger JL, Chow J, Miller MJ (2017) Human milk oligosaccharide consumption by probiotic and human-associated bifidobacteria and lactobacilli. J Dairy Sci 100(10): 7825-7833.

13. Yao Ang C, Sano M, Dan S, Leelakriangsak M, T ML, et al. (2020) Postbiotics Applications as Infectious Disease Control Agent in Aquaculture. Biocontrol Sci 25(1): 1-7.

14. Mosca F, Gianni ML, Rescigno M (2019) Can Postbiotics Represent a NewStrategy for NEC? Adv Exp Med Biol 1125: 37-45.

15. Zhaxi Y, Xiaoqin Meng, Wenhui Wang, Ling Wang, Zhuolin He, et al. (2020) Duan-Nai-An, A Yeast Probiotic, Improves Intestinal Mucosa Integrity and Immune Function in Weaned Piglets. Sci Rep 10(1): 4556.

16. Summers KL, Frey JF, Ramsay TG, Arfken AM (2019) The piglet mycobiome during the weaning transition: a pilot study1. J Anim Sci 97(7): 2889-2900.

17. Hung DY, Yeong Hsiang Cheng, Wei Jung Chen, Kuo Feng Hua, Arkadiusz Pietruszka, et al. (2019) Bacillus licheniformis-Fermented Products Reduce Diarrhea Incidence and Alter the Fecal Microbiota Community in Weaning Piglets. Animals (Basel) 9(12): 1145.

18. Levast B, Berri M, Wilson HL, Meurens F, Salmon H, et al. (2014) Development of gut immunoglobulin A production in piglet in response to innate and environmental factors. Dev Comp Immunol 44(1): 235244.

19. Cabrera RA, Lin X, Campbell JM, Moeser AJ, Odle J, et al. (2012) Influence of birth order, birth weight, colostrum and serum immunoglobulin $\mathrm{G}$ on neonatal piglet survival. J Anim Sci Biotechnol 3(1): 42.

20. Hu J (2018) Lactobacillus frumenti Facilitates Intestinal Epithelial Barrier Function Maintenance in Early-Weaned Piglets. Front Microbiol 9: 897.

21. Avonts L, De Vuyst L (2001) Antimicrobial potential of probiotic lactic acid bacteria. Meded Rijksuniv Gent Fak Landbouwkd Toegep Biol Wet $66(3 b): 543550$. 
ISSN: 2574-1241

DOI: 10.26717/BJSTR.2020.28.004584

Hsieh Hsun Ho. Biomed J Sci \& Tech Res

(C) (P) This work is licensed under Creative

Submission Link: https://biomedres.us/submit-manuscript.php

$\begin{array}{ll}\text { BIOMEDICAL } & \text { Assets of Publishing with us } \\ \text { RESEARCHES } & \text { - Global archiving of articles } \\ \text { - Immediate, unrestricted online access }\end{array}$

